\title{
EFEITO DO FOGO NA FLORÍSTICA E FITOSSOCIOLOGIA DE UM REMANESCENTE DE CERRADO SENSU STRICTO, TO
}

Effect of fire on floristics and phytosociology of a remaining from cerrado sensu

stricto, $T O$

Efecto del fuego en florística y fitosociología de un remanente de cerrado sensu stricto, $T O$

Bárbara Maria Martins Santos ${ }^{* 1}$, Bruno Aurélio Campos Aguiar ${ }^{1}$, Priscila Bezerra de Souza ${ }^{1}$.

${ }^{1}$ Laboratório de Sementes Florestais, Engenharia Florestal, Universidade Federal do Tocantins , Tocantins, Brasil. *Correspondência: Laboratório de Sementes Florestais, Universidade Federal do Tocantins, Rua Badejos Lote 7 , Chácaras 69/72-Zona Rural, Gurupi, Tocantins, Brasil. CEP:77.404-970. e-mail barbaramarttins1997@hotmail.com.

Artigo recebido em 08/03/2020 aprovado em 06/11/2020 publicado em 04/03/2021.

\section{RESUMO}

Objetivou-se conhecer a tolerância das espécies arbustivo-arbóreas ao fogo de um remanescente de cerrado sensu stricto em Gurupi-TO, após a passagem do fogo entre 2014 e 2018. Desenvolveu-se o estudo em um fragmento de cerrado sensu stricto na Reserva Legal da fazenda experimental da UFT, campus de Gurupi - TO. Foram inventariadas 05 parcelas de $5000 \mathrm{~m}^{2}(0,5$ ha) distribuídas de forma sistemática. No inventário realizado em 2014 encontrou-se 2.128 indivíduos, distribuídos em 110 espécies, 86 gêneros e 46 famílias, além das mortas em pé, já em 2018 foram quantificados 1.956 indivíduos, distribuídos em 107 espécies, 82 gêneros e 39 famílias, além do grupo das árvores mortas. No inventário realizado em 2018 ocorreu o desaparecimento de algumas espécies identificadas em 2014, tais como Kielmeyera speciosa, Connarus suberosus, Bauhinia rufa, Plathymenia reticulata, Swartzia multijuga, Agonandra brasiliensis e Styrax camporum. Em 2018 das 107 espécies amostradas, 53 espécies não houve mortalidade de nenhum indivíduo com a passagem do fogo sendo elas: Annona coriacea, Guatteria villosissima, Hirtella ciliata, Curatella americana, Dimorphandra mollis, Andira vermifuga, Acacia polyphylla, Byrsonima basiloba, Myrcia tomentosa, entre outras. Conclui-se que as espécies que não morreram após a passagem do fogo, podem ser indicadas para a recuperação de áreas.

Palavras-chave: Cerrado; Fogo; Resistencia das espécies.

\section{ABSTRACT}

The objective was to know the tolerance of shrub-tree species to the fire of a remnant of cerrado sensu stricto in Gurupi-TO, after the passage of fire between 2014 and 2018. The study was carried out in a fragment of cerrado sensu stricto in the Legal Reserve from the UFT experimental farm, campus of Gurupi - TO. 05 parcels of $5000 \mathrm{~m}^{2}$ (0.5 ha) were systematically distributed. In the inventory carried out in 2014, 2,128 individuals were found, distributed in 110 species, 86 genera and 46 families, in addition to the dead ones, in 2018, 1,956 individuals were quantified, distributed in 107 species, 82 genera and 39 families, in addition to the group of dead trees. In the inventory carried out in 2018, some species identified in 2014 disappeared, such as Kielmeyera speciosa, Connarus suberosus, Bauhinia rufa, Plathymenia reticulata, Swartzia multijuga, Agonandra brasiliensis and Styrax camporum. In 2018 of the 107 species sampled, 53 species did not have mortality from any individual with the passage of the fire being: Annona coriacea, Guatteria villosissima, Hirtella ciliata, Curatella americana, Dimorphandra mollis, Andira vermifuga, Acacia polyphylla, Byrsonima basiloba, Myrcia tomentosa, among others. It is concluded that the species that did not die after the passage of the fire, can be indicated for the recovery of areas.

Keywords: Cerrado; Fire; Species resistance 


\section{RESUMEN}

El objetivo era conocer la tolerancia de las especies de arbustos al fuego de un remanente de cerrado sensu stricto en Gurupi-TO, después del paso del fuego entre 2014 y 2018. El estudio se realizó en un fragmento de cerrado sensu stricto en la Reserva Legal de la granja experimental UFT, campus de Gurupi - TO. Se distribuyeron sistemáticamente 05 parcelas de $5000 \mathrm{~m}^{2}(0,5 \mathrm{ha})$. En el inventario realizado en 2014, se encontraron 2.128 individuos, distribuidos en 110 especies, 86 géneros y 46 familias, además de los muertos, en 2018 se cuantificaron 1.956 individuos, distribuidos en 107 especies, 82 géneros y 39 familias, además del grupo de árboles muertos En el inventario realizado en 2018, algunas especies identificadas en 2014 desaparecieron, como Kielmeyera speciosa, Connarus suberosus, Bauhinia rufa, Plathymenia reticulata, Swartzia multijuga, Agonandra brasiliensis y Styrax camporum. En 2018 de las 107 especies muestreadas, 53 especies no tuvieron mortalidad de ningún individuo con el paso del fuego: Annona coriacea, Guatteria villosissima, Hirtella ciliata, Curatella americana, Dimorphandra mollis, Andira vermifuga, Acacia polyphylla, Byrsonima basiloba, Myrcia tomentosa, entre otras. Se concluye que las especies que no murieron después del paso del incendio, pueden estar indicadas para la recuperación de áreas. Descriptores:Cerrado; Fuego; Resistencia a las especies

\section{INTRODUÇÃO}

O Cerrado é o segundo maior bioma da América do sul atrás apenas do bioma Amazônico, ocupando uma área de $2.036 .448 \mathrm{~km}^{2}$, cerca de $22 \%$ do território nacional, com alto nível de endemismo, abrigando 11.627 espécies de plantas nativas já catalogadas (BRASIL, 2019), também chamado de savana brasileira devido a sua similaridade fisionômica com as savanas africanas e australianas, foi definido por Mistry (2000) como um conjunto de ecossistemas estabelecidos por sua disponibilidade de nutrientes para as plantas, umidade, herbivoria e fogo, sendo assim constituído por comunidades dinâmicas.

A influência do fogo, em especial, ainda é um tema bem debatido na literatura, embora haja um consenso de que é um agente fundamental na estrutura e composição das comunidades vegetais (MASSI, 2017), como no cerrado brasileiro onde a maior parte de suas a fisionomias são tidas como ecossistemas dependentes do fogo (HARDESTY et al., 2005; PIVELLO, 2011), pois evoluíram sob sua influência e dele dependem para manter seus processos ecológicos, este pode ocorrer de forma natural, pois faz parte da ecologia deste bioma (COUTINHO, 2006), porém quando o fogo ocorre na estação seca do ano (normalmente por eventos antrópicos), pode atingir diretamente a diversidade biológica, ou seja, quanto mais seco o ambiente, maiores proporções terá a queimada, pois as gramíneas agem como combustível devido à quantidade de biomassa seca (GORGONEBARBOSA, 2016).

Dessa forma, durante a evolução, as espécies do Cerrado tornaram-se morfologicamente e fisiologicamente adaptadas ao fogo devido à sua frequência constante (BOND e WILGEN, 1996), é o que ocorre em uma das fisionomias do cerrado o sensu stricto que dispõe de árvores baixas e tortuosas, com ramificações assimétricas, folhas pilosas e firmes, pois as espécies, em sua maioria, possuem suberização (PAINE et al., 2010) alterando a densidade e a espessura do tronco, o que permite isolamento térmico das estruturas internas, juntamente com a capacidade de rebrota a partir de estruturas subterrâneas (rizomas), estas características são consideradas atributos pósfogo (COUTINHO, 1990). No entanto, queimadas muito intensas prejudicam plântulas ou indivíduos de menor porte que estão se estabelecendo (SATO e MIRANDA, 1996), e espécies expostas ao fogo regular podem desaparecer devido ao tempo de recuperação lento (AQUINO et al., 2007).

Nesta perspectiva, em áreas onde o fogo é frequente, uma progressiva redução da diversidade e simplificação da estrutura da comunidade pode ocorrer, tornando a fisionomia gradualmente mais 
aberta (HOFFMANN e MOREIRA, 2002). Dessa forma, a densidade do estrato arbóreo estaria determinada mais pela habilidade das espécies em sobreviver ao fogo e regenerar, do que pela própria competição pelos recursos ambientais (DURIGAN et al.,1994).

Considerando a riqueza de espécies presente no cerrado sensu stricto e o fogo como agente modulador desta vegetação, torna-se fundamental o desenvolvimento de estudos que possibilitem o melhor conhecimento da relação fogo/Bioma Cerrado com o objetivo de se conhecer a tolerância das espécies arbustivo-arbóreas ao fogo em uma remanescente de cerrado sensu stricto em Gurupi-TO, após a passagem do fogo entre 2014 e 2018.

\section{MATERIAIS E MÉTODOS}

$\mathrm{O}$ estudo foi desenvolvido em um fragmento de cerrado sensu stricto inserido na Reserva Legal da fazenda experimental da UFT, campus de Gurupi -

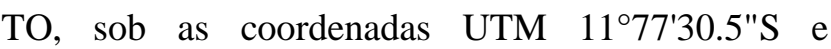
$49^{\circ} 05^{\prime} 62.9^{\prime \prime} \mathrm{W}$.

Foram inventariadas cinco parcelas de 5000 $\mathrm{m}^{2}$ (0,5 ha) distribuídas de forma sistemática, sendo distanciadas $10 \mathrm{~m}$ uma da outra. A primeira amostragem ocorreu em julho de 2014 (pré-fogo), quando todos os indivíduos com circunferência à altura do peito (CAP) maior ou igual que $10 \mathrm{~cm}$ a 1,30 $\mathrm{m}$ do solo foram medidos.

Dessa forma, foi realizada uma segunda amostragem do fragmento estudado em 2014 para avaliarmos a dinâmica do fragmento pós-fogo setembro de 2016 e novembro de 2017, onde verificou-se a dinâmica do fragmento quanto à sobrevivência ao fogo. Foram amostrados todos os indivíduos mortos em pé, considerando-se como critério a ausência de folhas e de gemas laterais, tronco oco e de fácil quebra.
A identificação taxonômica das espécies presentes na área de estudo de cerrado sensu stricto, tornou-se possível através de comparações com o material do herbário da UFT - Campus Porto Nacional, consulta à literatura, além de consultas a especialistas da área. O sistema de classificação adotado foi o "Angiosperm Phylogeny Group" (APG IV, 2016). A grafia e autoria dos binômios específicos e sinonímias foram confirmadas nas bases de dados "Lista de Espécies da Flora do Brasil" (REFLORA Herbário Virtual, 2020) - e IPNI - "International Plant Names Index" (IPNI, 2018).

Para o processamento dos dados coletados foi utilizado o programa Fitopac versão 2.1.2 (Shepperd, 2010). Onde foram determinados os parâmetros fitossociológicos como a somatória da área basal de cada espécie (AB - Área Basal), o número de indivíduos de cada espécie na área (DA - Densidade Absoluta), a relação entre o número de indivíduos de 56 uma determinada espécie pelo número total de indivíduos amostrados (DR - Densidade Relativa), a dominância absoluta de uma espécie pela área amostral ( $\mathrm{cm}^{2}$ ha-1) (DoA - Dominância Absoluta), a relação entre a área basal amostrada de uma determinada espécie e a área basal amostrada para todas as espécies expressa em porcentagem (DoR Dominância Relativa), o número de parcelas em que ocorre uma determinada espécie (FA - Frequência Absoluta), a porcentagem entre o número de parcelas de ocorrência da espécie em relação ao somatório de ocorrência de todas as espécies (FR - frequência relativa) e o Valor de Importância (VI) que sugere a representatividade geral de cada espécie na, comunidade e calculado a partir do somatório da densidade, dominância e frequência relativa seguida pela divisão por três. 


\section{RESULTADOS E DISCUSSÃO}

Nos inventários realizados no ano de 2014 e em 2018 a espécie que obteve destaque com maior número de indivíduos foi Tapirira guianensis pertencente à família Anacardiaceae.

No inventário realizado em 2014 encontrou-se 2.128 indivíduos com $\mathrm{CAP} \geq 10 \mathrm{~cm}$, distribuídos em 110 espécies, 86 gêneros e 46 famílias, além das mortas em pé. A média de altura da população foi de 7,23 metros e a área basal média de 10,88 $\mathrm{m}^{2} / \mathrm{ha}$ (Silva e Souza 2017), já em 2018 foram quantificados 1.956 indivíduos, distribuídos em 107 espécies, 82 gêneros e 39 famílias, além do grupo das árvores mortas em pé, com uma altura média da população de 7,25 metros e a área basal média de $10,17 \mathrm{~m}^{2} / \mathrm{ha}$.

Em 2014 as espécies que se destacaram em números de indivíduos foram: Tapirira guianensis (258), Protium heptaphyllum (227), Myrcia splendens (148), Vatairea macrocarpa (94), Guettarda viburnoides (84), Copaifera langsdorffii (76), Astronium fraxinifolium (70), Qualea parviflora (67), Grupo das mortas (66), Dilodendron bipinnatum (58), Anadenanthera peregrina (57) e Terminalia argentea (45).

As mesmas espécies se destacaram em 2018, porém com um menor número de indivíduos devido à passagem do fogo e como consequência a morte deles, assim aumentando o número do grupo dos indivíduos mortos, com os seguintes valores: Tapirira guianensis (233), Protium heptaphyllum (173), Grupo das mortas (158), Myrcia splendens (130), Vatairea macrocarpa (84), Guettarda viburnoides (76), Copaifera langsdorffii (71), Astronium fraxinifolium (66), Dilodendron bipinnatum (60), Qualea parviflora (58), Anadenanthera peregrina (53) e Terminalia argentea (44).

No inventário realizado em 2018 foi notado o desaparecimento de algumas espécies identificadas em 2014, tais como Kielmeyera speciosa, Connarus suberosus, Bauhinia rufa, Plathymenia reticulata, Swartzia multijuga, Agonandra brasiliensis e Styrax camporum, entretanto foi verificado no inventário de 2018, após a passagem do fogo, quatro novas espécies: Aspidosperma parviflorum, Bowdichia virgilioides, Myrocarpus, frondosus e Erythrina falcata.

Foi verificado que a ocorrência do fogo na área em 2015 e a recorrência do fogo em 2017 provocaram a morte de muitos indivíduos de várias espécies, 38 principalmente de indivíduos jovens e de pequeno porte fato este que corrobora com (MEWS et al., 2011).

Dessa forma, afirma-se que algumas famílias e espécies são tolerantes e não tolerantes a passagem do fogo. De acordo com SILVA et al. (2010), queimadas frequentes promovem uma alteração fenotípica de algumas famílias e espécies, agindo como filtro ambiental que consequentemente selecionam famílias e espécies capazes de sobreviver 57 e tolerar as condições abióticas derivadas da queima.

No inventário de 2018 dos 72 indivíduos que foram incluídos, o grupo das mortas obteve maior número de recrutamento com 19 indivíduos, já as espécies que ganharam novos indivíduos foram: Tapirira guianensis (17), Myrcia splendens (9), Dilodendron bipinnatum (4) e Copaifera langsdorffii (3). Os demais foram incluídos apenas com um indivíduo por espécie.

Foram identificadas 66 árvores mortas em pé e às mesmas foram incluídas dentro do grupo das mortas (SILVA e SOUZA 2017) no inventário de 2014. No inventário de 2018 foram identificadas 158 árvores mortas em pé, fato este que leva a crer que seja devido a fatores bióticos e abióticos (herbivoria, predação, falta de nutrientes e a passagem do fogo) presente na área de estudo em 2015 e 2017.

Das 111 espécies encontradas em ambos os levantamentos, de 2014 e 2018, 58 espécies foram quantificadas como mortas com um ou mais 
indivíduos supostamente por fatores bióticos, abióticos e a passagem do fogo, dessa forma foi possível observar que as espécies que se danificaram e morreram foram: Protium heptaphyllum com 31 indivíduos (17\% do total da espécie), Tapirira guianensis com 25 indivíduos (10\% da espécie), Myrcia splendens com 19 indivíduos (14\% da espécie), Vatairea macrocarpa com 11 indivíduos (13,4\% da espécie), Qualea multiflora com 7 indivíduos (17\% da espécie), Qualea parviflora com 7 indivíduos (12\% da espécie), Copaifera langsdorffii com 6 indivíduos (12\% da espécie), Tabebuia roseoalba com 6 indivíduos (12,5\% da espécie), entre outras espécies (Tabela 1)..

Tabela 1. Dinâmica (em número de indivíduos) das populações e das espécies lenhosas de uma área de cerrado sensu stricto amostrada na Reserva Legal da fazenda experimental da UFT, campus de Gurupi-TO no período de quatro anos 2014 e 2018 após a passagem do fogo.

\begin{tabular}{cccc}
\hline Espécies & $\begin{array}{c}\text { No de indivíduos } \\
\text { amostrados em 2014 }\end{array}$ & $\begin{array}{c}\text { No de indivíduos } \\
\text { mortos após o fogo } \\
\text { em 2018 }\end{array}$ & $\begin{array}{c}\text { \% de } \\
\text { mortes da } \\
\text { espécie }\end{array}$ \\
\hline Protium heptaphyllum (Aubl.) Marchand & 175 & 31 & 17 \\
Tapirira guianensis Aubl. & 258 & 25 & 10 \\
Myrcia splendens (Sw.) DC. & 129 & 19 & 14 \\
Vatairea macrocarpa (Benth) Ducke & 82 & 11 & 13,4 \\
Qualea multiflora Mart. & 41 & 7 & 17 \\
Qualea parviflora Mart. & 58 & 7 & 12 \\
Copaifera langsdorffii Desf. & 35 & 6 & 12 \\
Tabebuia roseo-alba (Ridl.) Sandwith. & 48 & 6 & 12,5 \\
Outras com mortalidade & 1155 & 46 & 3,98 \\
\hline
\end{tabular}

Segundo Ribeiro et al. (2012), o fogo aumenta a mortalidade de árvores, de plântulas e de indivíduos jovens (MEDEIROS e MIRANDA, 2005), e diminuindo o recrutamento, a altura da vegetação e ainda a taxa de crescimento de espécies lenhosas (SATO, 2005).

Das 107 espécies amostradas em 2018, em 53 espécies não houve mortalidade de nenhum indivíduo com a passagem do fogo em 2015 e 2017 sendo elas: Annona coriacea, Guatteria villosissima, Hirtella ciliata, Curatella americana, Dimorphandra mollis, Andira vermifuga, Acacia polyphylla, Byrsonima basiloba, Myrcia tomentosa, entre outras. Essas espécies podem ser consideradas resistentes ao fogo devido as suas características xeromórficas, súber grosso, troncos tortuosos, apresentando nenhuma morte de indivíduo da espécie.
Conforme observado por Felfili (1995) e Mews et al. (2011), as espécies que tiveram o maior número de indivíduos contabilizados como mortos apresentaram elevados recrutamentos no período. Segundo Felfili (1995) as espécies mais abundantes estão sujeitas as maiores taxas de mortalidade e recrutamento, justamente por ter elevada densidade, continuando esse padrão ao longo do tempo.

No presente estudo, o aparecimento e desaparecimento de espécies se limitaram na baixa abundância dos indivíduos $\leq 2$. Essas espécies de baixa abundância são classificadas como espécies raras (ASSUNÇÃO e FELFILI, 2004). Dados estes que corroboram com (AQUINO et al., 2007) em áreas de cerrado sensu stricto.

As alterações na composição florística que foram observadas na comunidade estudada estão 
relacionadas ao desaparecimento e surgimento de espécies, entre 2014 e 2018. Foram determinados 158 indivíduos mortos sendo incluídos 72 indivíduos.

O número de indivíduos e espécies novas foram inferiores ao da mortalidade, resultando em um balanço negativo para a comunidade, pois o fogo age como desbaste na vegetação lenhosa (FELFILI, 2000).

Os resultados demostram que o fogo exerceu um papel relevante na modificação da composição e estrutura da vegetação, extinguindo indivíduos, espécies e famílias da comunidade.

As mudanças que ocorreram na riqueza e composição florística, os valores de H' de $2014(3,65)$ e $2018(3,61)$ e equabilidade praticamente não mudaram entre os inventários $(2014=0,77$ e $2018=$ $0,77)$.

Tabela 2. Índice de Valor de Importância (IVI \%) em ordem decrescente das 14 famílias e do grupo das mortas nos dois anos inventariados 2014-2018.

\begin{tabular}{lcll}
\hline Famílias & IVI (\%) $\mathbf{2 0 1 4}$ & Famílias & IVI (\%) $\mathbf{2 0 1 8}$ \\
\hline Anacardiaceae & 12,97 & Anacardiaceae & 14,30 \\
Fabaceae & 11,36 & Fabaceae & 11,36 \\
Vochysiaceae & 7,12 & Vochysiaceae & 6,36 \\
Burseraceae & 6,55 & Myrtaceae & 5,80 \\
Myrtaceae & 5,85 & Burseraceae & 5,74 \\
Rubiaceae & 4,48 & Grupo das mortas & 4,86 \\
Sapindaceae & 4,56 & Sapindaceae & 59 \\
Malvaceae & 4,33 & Rubiaceae & 4,74 \\
Combretaceae & 3,58 & Combretaceae & 4,56 \\
Bignoniaceae & 3,09 & Bignoniaceae & 3,73 \\
Bombacaceae & 2,94 & Bombacaceae & 3,09 \\
Grupo das mortas & 2,67 & Apocynaceae & 2,89 \\
Tiliaceae & 2,46 & Chrysobalanaceae & 2,36 \\
Apocynaceae & 1,99 & Tiliaceae & 2,27 \\
Malpighiaceae & 1,96 & Polygonaceae & 2,25 \\
\hline
\end{tabular}

As famílias que apresentaram maiores números de indivíduos em 2018 após a passagem do fogo foram Anacardiaceae com 352 indivíduos (17,9\%), Burseraceae com 175 indivíduos $(8,9 \%)$, Vochysiaceae com 166 indivíduos (8,4\%), grupo das mortas com 158 indivíduos (8,0\%), Myrtaceae com 154 indivíduos (7,87\%), Fabaceae com 133 indivíduos
(6,80\%), Rubiaceae com 121 indivíduos (6,18\%) e Sapindaceae com 91 indivíduos (4,65\%) somando $68,89 \%$ do número total.

As famílias com os maiores números de espécies não mudaram de posição entre os dois levantamentos, merecendo destaque para as famílias 
Fabaceae, Malvaceae, Myrtaceae e Rubiaceae com 16, 7, 7 e 7 espécies, respectivamente.

As Famílias que representaram os maiores índices de valor de importância em 2018 somaram 48,29\% do total, sendo que a família Anacardiaceae foi a mais representativa com 14,30\%, seguida de Fabaceae com 11,36\%, Vochysiaceae com 6,35\%, Myrtaceae com 5,80\%, Burseraceae com 5,74\%, grupo das mortas com 4,79\% e Sapindaceae com 4,74\%, tendo um acréscimo em relação ao ano de 2014 de $29,44 \% 11,22 \%, 5 \%, 11 \%, 10,48 \%, 6,26 \%, 9,47 \%$ respectivamente do seu IVI (Tabela 2)

\section{CONCLUSÃO}

As alterações que foram observadas na área, possivelmente estão relacionadas a frequente passagem do fogo que teve um intervalo de dois anos de uma ocorrência para outra, favorecendo assim uma maior mortalidade dos indivíduos.

Com base nos resultados do presente estudo, as espécies que podem ser indicadas a uma possível recuperação de áreas onde ocorrem a passagem fogo, devem ter características xeromórficas como o suber grosso, troncos tortuosos, apresentando nenhuma morte de indivíduo da espécie, sendo elas: Annona coriacea, Guatteria villosissima, Hirtella ciliata, Curatella americana, Dimorphandra mollis, Andira vermifuga, Acacia polyphylla, Byrsonima basiloba, Myrcia tomentosa, entre outras.

As espécies que não podem ser indicadas a recomposição de áreas sujeitas a passagem de fogo são: Protium eptaphyllum, Tapirira guianensis, Myrcia splendens, Vatairea macrocarpa, Qualea multiflora, Qualea parviflora, Copaifera langsdorffii, Tabebuia roseo-alba.

\section{AGRADECIMENTO.}

Agradeço ao CNPq e ao grupo de pesquisa Serviços de Ecossistemas Florestais (SEF).
Todos os autores declararam não haver qualquer potencial conflito de interesses referente a este artigo.

\section{REFERÊNCIAS}

APG. Angiosperm Phylogeny Group. Na update of the Angiosperm Phylogeny Group classificatio for the ordens and families of flowering plants: APG IV. Botanical Journal the Linnean Society, n.181, p.120, 2016.

AQUINO, F. G.; WALTER, B. M. T.; RIBEIRO, J. F. Woody community dynamics in two fragments of "cerrado" strict sensu over a seven-year period (19952002), MA, Brazil. Revista Brasil. Bot. v. 30, n. 1, p. 113-121. 2007.

ASSUNÇÃO, S. L.; FELFILI, J. M. Fitossociologia de um fragmento de cerrado sensu stricto na APA do Paranóa, DF, Brasil. Revista Acta Botanica Brasilica, v.18, n., p.903-909, 2004.

BOND, W. J. e WILGEN, B. W. 1996 - Fire and plants. Chapman e Hall, New York. 263 p.

BRASIL. Ministério do Meio Ambiente (MMA). Bioma Cerrado - 2019. Disponível em: <http://www.mma.gov.br/biomas/cerrado>. Acesso em: 28 fev. 2019

COUTINHO, L.M. Fire in the ecology of the Brazilian cerrado. (Ed.). In: GOLDDAMMER, J.G. Fire in the Tropical Biota. Berlin, Springer-Verlag. 1990.p. 82105.

COUTINHO, L.M. O conceito de bioma. Acta Botanica Brasilica, Belo Horizonte, v.20, n.1, p.1-11, 2006.

DURIGAN, G.; LEITÃO FILHO, H. F.; RODRIGUES, R. R. Phytosocioloy and structure of a frequently burnt cerrado vegetation in SEBrazil. Flora, v. 189, n.2, p.153-160, 1994. 
FELFILI, J. M.; NOGUEIRA, P. E.; SILVA JÚNIOR, M. C.; MARINON, B. S.; DELITTI, W. B. C. Composição florística e fitossociologia do cerrado restrito do município de Água Boa, MT. Acta Botânica Brasílica, São Paulo, v. 16, n. 1, p. 103112 , 2002.

FELFILI, J. M.; VENTUROLI, F. Tópicos em análise de vegetação. Comunicações técnicas florestais, v. 2, n. 2 , p. $1-25,2000$.

FELFILI, J.M. Growth, recruitment and mortality in the Gama gallery forest in central Brazil over a sixyear period (1985-1991). J. Trop. Ecol. V.11 p. 6783, 1995.

GORGONE-BARBOSA, E. A relação entre fogo e uma gramínea invasora no Cerrado: $\mathrm{O}$ fogo pode ser utilizado como uma estratégia de controle. 2016. Tese de Doutorado. Thesis, Universidade Estadual Paulista "Júlio de Mesquita Filho" Google Scholar.

HARDESTY, J.; MYERS, R. \& FULKS, W. 2005. Fire, ecosystems, and people: a preliminary assessment of fire as a global conservation issue. The George Wright Forum, 22: 78-87.

HENRIQUES, R. P. B.; HAY, J. D. (2002), Patterns and dynamics of plant populations. In: Oliveira, $P$. S. and Marquis, R. J. (Eds.). The cerrados of Brazil: ecology and natural history of a neotropical savanna. New York: Columbia University Press. pp. 140-158.

HOFFMANN, W.A.; LUCATELLI, V.M.P.C.; SILVA, F.J.; AZEVEDO, I.N.C.; MARINHO, M.S.; ALBUQUERQUE, A.M.S.; LOPES, A.O.; MOREIRA, S.P. 2004. Impact of the invasive alien grass Melinis minutiflora at the savanna-forest ecotone in the Brazilian Cerrado. Diversity and Distributions. 10: $99-103$
IPNI - THE INTERNATIONAL PLANT NAMES INDEX. Search the Data: Plant Names. Disponível em: Acesso em: 23 out. 2018.

MASSI, K. G.; EUGENIO, C. U. O. and FRANCO, A. C. Reprodução pós-fogo de espécies do estrato herbáceo em área de transição Cerrado-Mata de Galeria no Distrito Federal, Brasil. Braz. J. Biol. 2017, vol. 77 , n. 4, p. 876-886.

MEWS, H. A.; SCHWANTES MARIMON, B.; MARACAHIPES, L.; FRANCZAK, D. D.; MARIMON-JUNIOR, B. H. Dinâmica da comunidade lenhosa de um Cerrado Típico na região Nordeste do Estado de Mato Grosso, Brasil. Biota Neotropica, v. 11, n. 1, 2011.

MEWS, H. A.; SCHWANTES MARIMON, B.; MARACAHIPES, L.; FRANCZAK, D. D.; MARIMON-JUNIOR, B. H. Dinâmica da comunidade lenhosa de um Cerrado Típico na região Nordeste do Estado de Mato Grosso, Brasil. Biota Neotropica, v. 11, n. 1, 2011.

MIRANDA, H. S.; ROCHA E SILVA, E. P.; MIRANDA, A. C. Comportamento do fogo em queimadas de campo sujo. Impactos de queimadas em áreas de cerrado e restinga, p. 1-10, 1996.

MIRANDA, H.S.; SATO, M.N. Efeitos do fogo na vegetação lenhosa do Cerrado. In: SCARIOT, A.; SOUSA-SILVA, J. C.; FELFILI, J. M. (Org.). Cerrado: ecologia, biodiversidade e conservação. Brasília: MMA, 2005. p. 95-103.

PAINE, C. E. T.; COURTOIS, E. A.; PATINO, S.; SARNIENTO, C.; BARALOTO, C.Functional explanations for variations in bark thickness in tropical rain forest trees. Func. ecol. v. 24, n. 6, p. $1202-1210$. 2010. 
PIVELLO, V.R. 2011. The use of fire in Brazil: past and present. Fire Ecology, 7: 24-39.

RIBEIRO, M.N.; SANCHEZ, M.; PEDRONI, F.; PEIXOTO, K.S. Fogo e dinâmica da comunidade lenhosa em cerrado sentido restrito, Barra do Garças, Mato Grosso. Acta Botânica Brasilica, v. 26, n.1, p.203-217, 2012.

SATO, M. N.; MIRANDA, H. S. 1996. Mortalidade de plantas lenhosas do cerrado sensu stricto submetidas a diferentes regimes de queimada. In: MIRANDA, H. S.; SATO, M.N.; MIRANDA, H.S.;
MAIA, J.M.F. O fogo e o estrato arbóreo do Cerrado: efeitos imediatos e de longo prazo. In: MIRANDA, H.S. (Org.). Efeitos do regime do fogo sobre a estrutura de comunidade de Cerrado: Projeto fogo. Brasília: IBAMA, 2010. p.77-91.

SHEPHERD, G. J. Fitopac 2: manual do usuário. Campinas: Unicamp, 2010. 91p.

SILVA, G. O.; SOUZA, P. B. FITOSSOCIOLOGIA E ESTRUTURA DIAMÉTRICA DE UM FRAGMENTO DE CERRADO SENSU STRICTO, GURUPI-TO. Desafios, v. 3, p. 22-29, 2017. 